\title{
XXX.
}

Aus der medizinischen Universitäts-Poliklinik zu Freiburg i. Br.

(Direktor: Prof. Dr. K. Ziegler).

\section{Ueber den Einfluss des Quecksilberquarzlampenlichts auf die Resistenz der roten Blutkörperchen gegenüber hypotonischen Kochsalzlösungen.}

\author{
Von
}

\section{Konrad von Rohden.}

Die grosse Ausbreitung des Bergsportes in den letzten Jahrzehnten und die gleichzeitig dabei in grösseren Höhen oft beobachteten merkwürdigen körperlichen Erscheinungen, die man unter dem Namen Bergkrankheit zusammenfasst, vor allem aber die glänzenden Erfolge, die bei der Behandlung der Tuberkulose im Höhenklima erzielt wurden, waren der Anstoss, dass sich die Wissenschaft mit der Erforschung des Höhenklimas zu befassen begann. Nach mühevollen Untersuchungen, die auf Expeditionen in die Alpen oder in eigens dazu in grossen Höhen im Hochgebirge eingerichteten Laboratorien angestellt worden waren, ist man jetzt nach vielen widersprechenden Ergebnissen zu einigen sicheren Resultaten über die Einwirkung des Höhenklimas auf den menschlichen Organismus gekommen.

Vor allem ist jetzt die Frage der Beeinflussung der Erythrozytenzahlen und des Hämoglobins als geklärt anzusehen. Nachdem die von Egger u. a. behauptete grosse Vermehrung derselben von späteren Untersuchern, besonders Bürker, Stäubli, Douglas nicht wieder gefunden werden konnte, haben jetzt Cohnheim und Weber sowie Laquer die Frage dahin entschieden, dass bei kurzem Aufenthalt in der Höhe eine wesentliche Zunahme der Erythrozytenzahlen und des Hämoglobins nicht, bei längerem dagegen deutlich in Erscheinung tritt.

Die Leukozytenzahl hält sich nach Stäubli und Baer-Engelsmann innerhalb der für das Tiefland geltenden Zahlen. Jedoch ist für das Höhenklima eine Zunahme der Lymphozyten, sowohl prozentual als absolut, nachgewiesen bei gleichzeitiger Verminderung der veutrophilen Leukozyten (Baer-Engelsmann).

Bürker gibt an, in der Höhe erfolge eine beschleunigte Gerinnung des Blutes. Nach Deetjen beruht das auf einer beschleunigten Kohlensäureverdarnpfung, die bei geringem Barometerdruck eintritt.

Der Süuregehalt des Blutes ist in grösseren Höhen grösser als in der Ebene (Galeotti, Aggazotti u. a.).

Auch bezüglich des Einflusses der Atmung, der Sauerstoffversorgung und des Gasaustausches des Körpers im Hochgebirgsklima sind wir jetzt 
genauer unterrichtet. Nach Durig ist die Atemfrequenz oft gesteigert, aber nicht regelmässig. Bei einigen Menschen kommt aber auch Verminderung vor. Die Tiefe der Atmung ist ganz allgemein vermehrt, eine Tatsache, die dank der besseren Sauerstoffversorgung und der Lungengymnastik in klinisch-therapeutischer Hinsicht von grosser Bedeutung ist. In grösseren Höhen wird die Atmung periodisch (Mosso, Haldane, Douglas).

Die verminderte Sauerstoffzufuhr zum Körper infolge des verminderten $\mathrm{O}_{2}$-Druckes in der Höhe ist wohl der Grund zu einem vermehrten Stoffumsatz, sowohl bei Ruhe wie anch bei Muskelarbeit. Nach Durig, Zuntz, zeigen alle Versuchspersonen einen deutlich verstärkten $\mathrm{O}_{2}$-Verbrauch und eine entsprechend vermehrte Kohlensäureproduktion. Der Respirationsquotient ist nicht verändert. Der sinkende $\mathrm{O}_{2}$-Druck führt also zu einer vermehrten Verbrennung im Körper.

Mosso, Durig u. a. beobachteten in grossen Höhen eine Pulsbeschleunigung, die in mittleren Höhen allerdings nur angedeutet ist. Der Blutdruck blieb immer unverändert.

Durig, Zuntz und Neuberg stellten eine grosse Steigerung der Stickstoffausscheidung und in geringerem Masse der Chlorausscheidung durch die Hant fest, eine Tatsache, die sie auf die hochgradige Trockenheit der Luft zurückführen.

Jaquet und Stähelin machten Versuche über den Stoffwechsel und fanden in der Höhe, nachdem ein geringer Eiweissverlust vorangegangen war, starken Eiweissansatz, und zwar Ansatz vom Organeiweiss.

Diese letztere Beobachtung und die - nicht erhebliche - Zunahme der Erythrozytenzahl und des Hämoglobins, sowie die Vertiefung der Atmung allein können aber noch nicht die günstigen Wirkungen des Höhenklimas vollständig erklären. Die Mitteilungen Bernhard's und Rollier's über ihre glänzenden Resultate bei der Sonnenbehandlung der chirurgischen Tuberkulose lenkten nun die allgemeine Aufmerksamkeit auf die Strahlenwirkung. Rollier selber schrieb aus einer Hypothese heraus den ultravioletten Strahlen diese hervorragende therapeutische Wirksamkeit zu. Dass dieselben besondere Bedeutung haben, schloss er aus der Beobachtung, dass der Grad der Pigmentbildung ein Indikator für die Aussichten der Heilung war. Und dass die ultravioletten Strahlen Pigment hervorrufen, weiss man seit Unna, Widmark und vor allem Finsen. Doch sagt er selber, dass es sich noch nicht scharf auseinander halten lässt, was bei der Heilung der Tuberkulose lokale Hyperämie, bakterizide, physikalische und chemische Wirkung der Sonnenstrahlen und was die tonisierende Wirkung" der Höhenluft bedeutet. Die Sehwierigkeit der Beurteilung ergibt sich aus der Kompliziertheit des Begriffs Höhenklima. Ausser Totaldruck, $\mathrm{O}_{2}$-Druck, Temperatur, Feuchtigkeit, Luftbewegung und der eigentlichen Strahlenwirkung kommen noch Ozongehalt, Ionisierung und Radioaktivität der Atmosphäre in Betracht, vielleicht sogar Faktoren, die wir noch gar nicht kennen.

Hasselbach and Lindhardt versuchten nun experimentell den Einfluss der verschiedenen Faktoren klarzustellen und zwar durch Einrichtung eines pneumatischen Kabinetts, in dem die einzelnen in Betracht 
kommenden Faktoren einzeln oder in Kombination miteinander in ihrer Wirkung auf den menschlichen Organismus erprobt werden konnten.

Sie kommen zu dem Resultat, dass die hauptsächlichsten, schon bekannten physiologischen Wirkungon des Höhenklimas, die Atmung und den Kreislauf betreffend, der Luftverdünnung, d. h. der Herabsetzung des Sauerstoffdruckes allein zu verdanken sind, wie man es ja auch schon vorher angenommen hat. Vor allem beruhe auf ihm auch die Steigerung der Erythrozytenzahlen und des Hämoglobingehaltes, was auch schon von Scheumann und Rosenquist, Kuhn, und bei Fliegern (E. Meyer, Kaulen) festgestellt worden war.

Früher schon hatten sie nachgewiesen, dass das Licht der Kohlenbogenlampe eine Verlangsamung und Vertiefung der Atmung hervorrufe, falls ein universelles Lichterythem auftritt. Das ist also als eine Wirkung der ultravioletten Strahlen zu betrachten, an denen die Kohlenbogenlampe reich ist. Die Entdeckung des Quecksilherdampflichtes vor allem und seine Anwendung in der Kromayer'schen Lampe und neuerdings in der „künstlichen Höhensonne", die ja so gut wie nur ultraviolette Strahlen aussendet, ermöglichte es, die Wirkung der ultravioletten Strahlen auf den Organismus für sich gesondert in eingehender Weise zu klären, so dass man die Wirkung der Strahlen und der sonstigen Faktoren des Höhenklimas trennen konnte und chuf diesem Wege vielleicht gesicherte Daten über den günstigen Einfluss desselben bekam, für dessen Erklärung man bis dahin nur auf vage Vermutungen angewiesen war.

Quincke, Hertel, Bering bewiesen die Steigerung der Oxydationskraft der Zelle, Pinkussohn eine Beeinflussung des Stoffwechsels, Bering und Meyer die fördernde Wirkung auf die Oxydationsfermente, Neuberg eine Spaltung der Eiweisskörper, Fette und Kohlehydrate, Bach eine Blutdruckverminderung.

Zahlreich sind anch die Untersuchungen, die sich auf den Einfluss der ultravioletten Strahlen auf das Blut beziehen. Nach vielen, sich widersprechenden Ergebnissen ist es jetzt als sicher anzusehen, dass eine Vermehrung der roten Blutkörperchen und des Hämoglobingehaltes jedenfalls nicht eintritt: Jedoch tritt eine Abnahme der Leukozyten ein, bei gleichzeitiger Lymphozytose (Wernscheid). Waltscheff allerdings berichtet von einer Vermehrung der Leukozyten, besonders der Nentrophilen. Hausmann und Meyerhofer finden einen hemmenden Einfluss des Quarzlampenlichtes auf die Blutgerinnung.

Dreyer und Hansen, Schmidt-Nielsen, Hasselbach studierten den Einfluss des Quecksilberlichtes auf das Blut, und zwar in vitro, d. h. sie brachten Blutkörperchenaufschwemmungen in Quarzkammern und belichteten dann. Sie fanden, dass bei starken Bestrahlungen Hämolyse der Blutkörperchen eintrat. Hasselbach stellte gleichzeitig die Umwandlung des Blutfarbstoffes in Methämoglobin fest, welch letzteres sich wieder weiter zersetzt, unter anderem in Hämatin.

Schmidt-Nielsen berichtet als erster über eine Beeinflussung der Resistenz der Erythrozyten gegenüber hypotonischen Kochsalzlösungen. Er fand, dass die Hämolyse bei belichteten Blutkörperchen schon bei 0,9 proz. NaCl-Lösungen begaun, wäbrend sie bei unbelichteten erst bei 
einem Salzgehalt von 0,6 pCt. eintrat. Die belichteten Erythrozyten waren also weniger resistent. Eine vollständige Hämolyse trat jedoch nicht ein. Hasselbach führte das später auf die zu dicken Wände der Quarzkammer zurück.

Diese schwer schädigenden Wirkungen sind an sich nicht weiter auffallend, haben doch alle Autoren bei ihren Untersuchungen bei schwacher Einwirkung des Quecksilberlichts auf die Zelle zunäehst eine Förderung der biologischen Vorgänge, erst bei stärkerer eine Schädigung und zuletzt Abtötung der Zelle gefunden ( $z$. B. Bering und Levy). In dieser starken Konzentration vermögen die ultravioletten Strahlen wegen ihrer geringen Durchdringungsfähigkeit auf den Organismus, das Blut und die inneren Organe nicht zu wirken. Sie werden nach Hasselbach schon in der Epidermis absorbiert. Zndem verfügt der Organismus über eine Schutzwirkung, nämlich das Pigment, das nach Rollier die stark wirkenden kurzwelligen in die schwächeren langwelligen Strahlen umwandelt. Und doch geben einige Beobachtungen Aulass zu der Vermutung, dass so ganz einfach die Dinge nicht liegen. Finmal haben Hausmann und Mayerhofer zwei Fälle angefübrt, wo es bei zwei Kindern während der Lichttherapie zum Auftreten von reichlichem Blutfarbstoff im Harn ohne Nephritis kam. Ob dies als sicherc Strahlenwirkung zu betrachten ist, lässt sich nicht entscheiden. Aber die Möglichkeit einer schädigenden Wirkung der Strahlen liegt doch vor. Zum anderen Mal hat M. Levy über ausgedehnte Veränderungen an der Milz von Mäusen nach Bestrahlung mit ultraviolettem Licht berichtet. Sie fand makroskopisch Vergrösserung des Organs um das zwei- bis dreifache, mikroskopisch eine enorme Hyperamie der Pulpa und extravasale Blutungen, eine Umlagerung der Follikel mit mächtigen Massen eines kernarmen hyalinen Gewebes und eine bisweilen sehr beträchtliche Vermehrung der Riesenzellen (Megakaryozyten). Wie diese Schüdigung zustande gekommen ist, ist noch nicht recht erklärbar, aber immerhin sind das doch wohl Folgen eines tiefer wirkenden Einflusses der ultravioletten Strahlen. Ob diese Wirkung mit den kurzwelligen Strablen des Hochgebirges zu erreichen wäre, bleibe dahingestellt. Etwas zo woit wird es jedoch gegangen sein, wenn Jesionek, von den Wirkungen der Strablen in Höhe von 1500 bis $1800 \mathrm{~m}$ sprechend, sagt: "Wir besitzen nicht die Spur eines positiven Anhaltspunktes dafür, dass in diesen Höhenlagen das Blut des Menschen in die Lage kommt, chemische Strahien der Sonne zu absorbieren. Im Blut lassen sich keine Vorgänge oder Veränderungen nachweisen, welche mit Bestimmtheit auf derartige direkte Absorptionsvorgänge schliessen liessen."

Es bedarf also noch weiterer Arbeiten, um die Einwirkung der Sonnenstrahlen, insbesondere die des kurzweiligen T'eils ihres Spektrums, zu klären.

Wanner hat nun die Untersuchungen auf ein bis dahin noch nicht begangenes Gebiet ausgedehnt, nämlich auf den Einfluss des Höhenklimas anf die Widerstandskraft der Erythrozyten gegen hypotonische Kochsalzlösungen. Eine grosse Anzahl von gesunden oder annähernd gesunden Kindern, die für sechs Wochen rom Flaehland ins Gebirge auf 
$1500 \mathrm{~m}$ als Ferienkolonisten gesandt wurden, gaben ihm ein geeignetes Untersuchungsmaterial. Er untersuchte nach der nach Hamburger benannten makroskopischen sogenannten Blutkörperchenmethode die Resistenz der Erythrozyten gegenüber hypotonischen Kochsalzlösungen, mit einer von Ribierres angegebenen Modifikation. Er stellte sich in einer Anzahl von Reagenzröhrchen eine Reihe von $\mathrm{NaCl}$-Lösungen versehiedener Konzentration her, und $z$ war von 0,50 pCt. fallend mit cinem jeweiligen Konzentrationsunterschied von $0,02 \mathrm{pCt}$. In eigens dazu erfundener Mischpipette wurde ein durch einen Lanzettstisch gewonnener Blutstropfen aufgenommen, die Flüssigkeit je eines Röhrchens nachgesaugt und dann ausgeblasen. Nach fünf Minuten zentrifugierte er und las dann den Beginn der Hämolyse ab und verzeichnete beginnende deutliche, sehr deutliche sowie vollständige Hämolyse. Er fand nun die ihn überraschende Tatsache, dass nach ihrer Rückkehr aus der Höhe bei 29 pCt. der Kinder die Resistenz unverändert war, während sie bei 65 pCt. der Kinder vermindert war.

Wie war das zu erklären? Sollte das wirklich eine schädigende Wirkung des Hochgebirgsklimas sein, insbesondere der starken Strahlung? Nach den Beobachtungen, die Schmidt-Nielsen gemacht hatte (siehe oben), lag es ja zunächst nahe, daran zu denken. Doch sind die Kinder nicht einer längeren ausdrücklichen, auch nicht, wio sie Rollier in Leysin verwendet, Ganzbestrahlung des Körpers ausgesetzt gewesen. Wanner findet eine Erklärung dadurch, dass or sagt, die beim Aufenthalt im Hochgebirge zahlreich neugebildeten jungen Erythrozyten, die aus dem Knochenmark ins Blut kommen, sind weniger resistent als die älteren. Andere Autoren, die sich mit der Resistenz der Erythrozyten befassten, wie Sattler, Krasny, geben dagegen an, dass gerade die später ins Blut gelieferten Blutkörperchen resistenter sind als die schon länger kursierenden abgenutzten debilen Formen, die nach einer Lebensdauer von vier Wochen dem Untergang verfallen sind. Nach Itami und Pratt ist das Alter der Erythrozyten überhaupt nicht massgebend.

Eine Erklärung für die von Wanner angegebene Resistenzverminderung zu finden, ist noch nicht möglich. Ist es ja noch nicht einmal deutlich, welcher von den vielen Faktoren, die das Hochgebirgsklima im ganzen eben ausmachen, die eigentliche Ursache ist. Die Möglichkeit, dass diese Wirkung auf das Blut auf der intensiven Strahlung beruht, insbesondere der chemisch wirksamen ultravioletten Strahlung im Hochgebirge, die ja bekanntermassen erheblich viel stärker ist, als in der Ebene (Dorno), ist nicht von der Hand zu weisen.

Daher habe ich es unternommen, die Wirkung der ultravioletten Strahlen, wie sie dio Quecksilberdampquarzlampe fast ausschliesslich ausstrahlt, auf dic Resistenz der Erythrozyten gegenüber hypotonischen Kochsalzlösungen im menschlichen Organismus erneut zu untersuchen.

Als Lichtquelle diente die Bach'sche "künstliche Höhonsonne" und zwar wurden immer gleichzeitig zwei Lampen verwandt, um eine Ganzbestrahlung des Körpers zu ermöglichen. Die Dauer der Bestrahlung wurde langsam gesteigert, beginnend mit 4 Minuten, 2 Minuten für die Vorder- und zwei Minuten für die Rückseite des Patienten. Je nach seiner Empfindlichkeit konnte mehr oder weniger schnell auf acht 
Minuten, zwölf Minuten usw. bis zu einer Stunde durchschnittlich gegangen werden. Die Lampen hatten einen regelmässigen Abstand von $1 \mathrm{~m}$. Zwischen den einzelnen Bestrahlungen lag gewöhnlich ein Zwischenraum von zwei bis drei Tagen, so dass stärkere Errytheme vermieden wurden. Doch trat eine leichte Pigmentierung in der Regel ein.

Vor Beginn der Bestrahlung wurde bei jeder Versuchsperson regelmässig eine Blutuntersuchung gemacht, dann während der Bestrahlung ein- oder zwcimal; oft auch nach Beendigung derselben noch einmal.

Für die Ausführung der Resistenzbestimmung standen eine Anzahl von Methoden zur Auswahl. In den letzten Jahren vor dem Kriege war viel an ihrer Verbesserung und Vereinfachung gearbeitet worden. Es würde zu weit führen auf dieselben alle einzugehen. Charlton, der noch als Letzter diese Frage behandelt hat, kommt zu dem Schluss, dass Anspruch auf alleinige Berechtigung keine der z. Z. üblichen Methoden hat. Ich wandte die Methode von Hamburger an, wie es auch schon Wanner getan hatte.

Mittels Venenpunktion wurde aus der Arrnvene das Blut entnommen und zur Verhinderung der Gerinnung mit konzentrierter Glaubersalzlösung vermischt, und zwar im Verhältnis ein Teil Glaubersalz auf drei T'eile Blut. In eine Reihe von Reagenzgläschen kam je $1 \mathrm{ccm}$ von einer $0,60 \mathrm{pCt}$. bis zu $0,14 \mathrm{pCt}$. abnehmenden Kochsalzlösung, deren Konzentration von Röhrehen zu Röhrehen um $0,02 \mathrm{pCt}$. ficl. Hergestellt wurden die Lösungen aus einer 1 proz. Stammlösung und destilliertem Wasser durch Abmessung mit der Pipette. In jede dieser versehiedenen

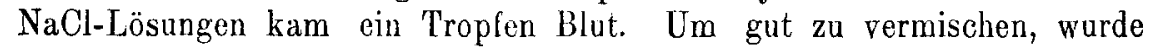
danach sanft geschüttelt. Die Mischung blieb dann bei Zimmertemperatur 2 Stunden stehen. Nach dieser Zeit hatten sich die Blutkörperchen gewöhnlich abgesetzt, so dass jetzt eine Ablesung erfolgen konnte. Nach 24 Stunden wurde noch einmal eine Kontrollablesung gemacht, die in der Regel mit der ersten gut übereinstimmte. Un das subjektive Moment bei der Beurteilung der Farbenunterschiede möglichst auszuschalten, wurde die Ablesung tunlichst von zwei Beobachtern ausgeführt.

Ich bin mir klar darüber, dass meine, von den sonstigen Methoden abweichende Behandlungsart, insbesondere, was die Verwendung von Glaubersalz betrifft, keine Vergleichresultate mit den Ergebnissen anderer Untersucher geben kann. Von grosser Bedeutung dürte das nicht sein, da auch die Ergobnisse der meisten Untersucher, wegen der vielen verschiedenen von ihnen angewandten Methoden, ebensowenig ohne weiteres untereinander vergleichbar sind. Die Behandlung der Erythrozyten mit der konzentrierten Glaubersalzlösung muss ihre Resistenz zweifellos beträchtlich beeinflussen. So sind uns auch sonst eine Menge von Momenten bekannt, die dieselben verändern können. Z. B. der Alkohol, Chinin, Kohlensïuregehalt, Temperaturen und einige Diuretika. Weiterhin wird auch die Konzentration der Salzlösungen gewissermassen eine höhere, so dass die gefundenen Werte durch diese Umstände erheblich anders ausfallen, als die der anderen Untersucher. Aber unter sich sind die Werte durchaus vergleichbar, da jedesmal vor der Bestrahlung der normale Resistenzwert für die betreffende Versuchsperson festgestellt wurde, auf 
den dann alle während oder nach der Bestrahlung auf genau dieselbe Weise gewonnenen Resultate bezogen werden kounten.

Als Versuchspersonen. standen einmal Patienten der Poliklinik zur Verfügung, die zu therapeutischen Zwecken bestrahlt wurden. Auf der anderen Seite war es jedoch auch wünschenswert, an Gesunden die Resultate nachzuprüfen. Es haben sich zu diesem Zwecke einige Kollegen in liebenswürdiger Weise zur Verfügung gestellt.

Ich bespreche zunächst diejenigen, die an den Patienten gewonnen wurden.

Fall 1. Fran E. K., 41 Jahre, leidet an klimakterischen Beschwerden. Ausserdem besteht eine Leukopenie bei ihr. Sie bekommt innerlich Arsenferratose.

Die Blutuntersuchung ergab folgendes Resultat:

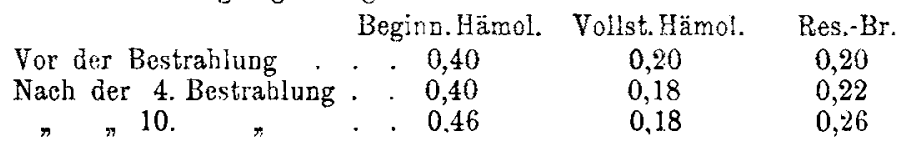

Es ist daraus zu sehen, dass die sogenannte von Mosso eingeführte Maximumresistenz, d. h. die Kochsalzkonzentration, bei der sämtliche, auch die resistentesten Erythrozyten aufgelöst sind und ihr Hämoglobin abgegeben haben, kleiner geworden ist. Denn die roten Blutkörperchen werden nach der Bestrahlung erst in noch schwächeren Kochsalzlösungen aufgelöst oder, anders ausgedrückt, sie widerstehen jetzt destilliertem Wasser besser als vorher. Sie haben eine erhöhte Widerstandskralt.

Um den irreführenden Umstand zu vermeiden, dass bei Lrhöhung der Resistenz die Maximumresistenz kleiner wird, hatten Hamburger u. a. schon vorgeschlagen die Maximumresistenz mit reziprokem Wert anzugeben, doch hat sich dies merkwürdigerweise nicht einbürgern können.

Aber auch dic von v. Limbeck eingeführte llinimumresistenz, also die NaCl-Konzentration, bei der die ersten Erythrozyten, nämlich die widerstandsschwächsten ihr Hämoglobin abgeben, ist verändert. Sie ist von 0,40 auf 0,46 gestiegen. Es sind jetzt nach der Bestrahlung rote Blutkörperchen vorhanden, die in stärkeren als durch die erste Untersuchung für die betreffende Versuchsperson als normal geltenden Konzentrationen schon ihren Farbstoff abgeben. Sie haben also gewissermassen eine Abschwächung ihrer Resistenz erfahren.

Welche von beiden Werten soll man nun die grössere Bedeutung beilegen. Von den meisten Untersuchern, die sich mit der Erythrozytenresistenz beschäftigten, wurde, wic Cohnreich sagt, „leider nicht die Resistenzbreite, sondern wegen ihrer leichten Auffindbarkeit die Minimumresistenz bestimmt, obwohl deren zweifelhafter Wert mehrfach betont worden war. (Itami und Pratt, Rosenthal, Ribierre, Janowsky, Lang.) Die Minimumresistenz ist nämlich in hohem Masse schon bei geringen Schwankungen der Einwirkungsdauer hypotonischer Salzlösungen unbeständig und verschieblich, die die Maximumresistenz kaum beeinflussen." Daher habe auch ich bei meinen Resultaten das grössere Gewicht auf die Maximumresistenz gelegt.

Gleichzeitig habe ich jedesmal den Wert für die Resistenzbreite, das ist das Konzentrationsgebiet zwischen Maximum- und Minimumresistenz, angegeben. Sie ist hier vergrössert. 
Ist es nun sicher die Bestrahlung, die diese Erhöhung der Widerstandskraft der Erythrozyten hervorgerufen hat oder können noch vielleicht andere Momente als Ursache dafür in Frage kommen? So z. B. Arsen? Strasser und Neumann behaupten nämlich, Arsen erzeuge eine echte Erhöhung der Resistenz. 1916 hat Thiele diese Frage einer eingehenden Nachprüfung unterzogen und kommt zu dem Schluss, dass die Resistenz gegenüber hämolytischen Substanzen, wie destilliertes Wasser, Zyklamin und Glykocholate nicht gesteigert wird.

Strasser und Neumann geben weiterhin an, der Hämoglobirgehalt sei von Einfluss, bei dessen Steigen die Werte der Isotonie auch in die Höhe gingen. Nach Itami und Pratt ist das jedoch nicht der Fall. Sie konnten zwischen Hämoglobin und Resistenz kein proportionales Verhältnis finden. Die Vermehrung des Hämoglobingehalts ist hier auch garnicht zu erwarten, da nach den übereinstimmenden Befunden der letzten Untersucher, vor allem nach den eingehenden Untersuchungen von Königsfeld, der Hämoglobingehalt durch die ultravioletten Strahlen nicht beeinflusst wird. Somit kann auch eine etwa durch den Gebrauch von Eisen hervorgerufene Hämoglobinvermehrung unberücksichtigt gelassen werden. Es ist daher als sicher anzunehmen, dass die Resistenzerhöhung eine Folge der Bestrahlung ist.

Fall 2. Frau F. M., 39 Jahre, leidet an Lungentuberkulose. Sie bekommt Arsen plus Eisen verabreicht.

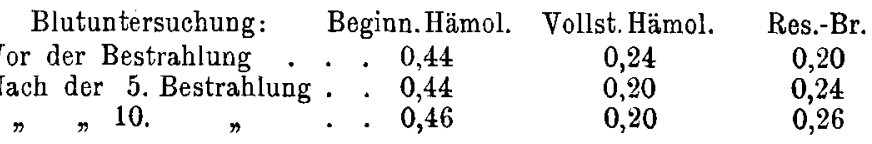

Aueh hier ist also wieder eine Vergrösserung der Resistenzbreite eingetreten, die schon nach der 5. Bestrahlung von 2 mal 10 Minuten Dauer deutlich ist.

Der Einwand, es könnte vielleicht der Verlauf der Tuberkulose und nicht die Bestrahlung die Ursache dafür sein, kann mit den Untersuchungsergebnissen Weihrauch's entkräftet werden, der angibt, dass in nur 2,9 pCt. aller Tuberkulosen eine Resistenzverminderung zu finden war. Prognostischen Wert habe dies Ergebnis nicht, da auch bei sehweren Fällen die Resistenz unverändert blieb. Allein schon der kurze Zwischenraum zwischen den beiden Untersuchungen, 10.10. bis 27. 10., macht es unwahrscheinlich, dass eine bedeutsame Veränderung des Krankheitsprozesses, die als Ursache der Resistenzvermehrung aufgefasst werden könnte, hat eintreten können.

Fall 3. Frl. H., 23 Jahre, leidet an starkern Basedow.

Blutuntersuchung: Beginn.Hämol. Vollst.Hämol. Res.-Br.

Vor der Bestrablung . . . $0,40 \quad 0,20 \quad 0,20$

Nach der 3. Bestrahlung . . $0,40 \quad 0,20 \quad 0,20$

$\Rightarrow \begin{array}{llllll}n & 10.0,46 & 0,14 & 0,32\end{array}$

Die Erhöhung der Resistenz ist offensichtlich.

Fall 4. Frau W., 24 Jahre, leidet an Lungenspitzenkatarrb.

Blutuntersuchung: Beginn. Hämol. Vollst. Hämol. Res.·Br.

Vor der Bestrahlung . . . $0,40 \quad 0,20 \quad 0,20$

Nach der 4. Bestrahlung : . $0,42 \quad 0,16 \quad 0,26$

Zeitschrift f. exp. Pathologie u. Therapie. 21. Bd. 3. H. 
Fall 5. Herr S., Student. Abgeheilte Pleuritis mit Schwartenbildung. Blutuntersuchung: Beginn. Hämol. Vollst.Hämal. Res.-Br. Vor der Bestrahlung . . . $0,44 \quad 0,18 \quad 0,26$ Nach der 7. Bestrahlung . . $0,48 \quad 0,16 \quad 0,32$

Fall 6. Herr R., Soldat, 21 Jahre, leidet an Iungen- und Bauchfelltuberkulose. Blutuntersuchung: Beginn. Hämol. Volist. Hämol. Res.-Br.

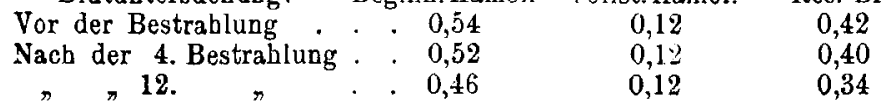

Hier ist keine Veränderung der Maximunresistenz sichtbar. Dieselbe ist an sich abnorm niedrig, was sich wohl aus einer sehon vor 14 Tagen stattgehabten Lichttherapie oder die mässige chronische Anämie des Patienten erklärt. Auffällig ist auch das Fallen der Werte für die Minimumresistenz.

Fall 7. St. M., Mädchen, 9 Jahre, leidet an tuberkulösen Drüsen: Sie bekommt Phosphor-Lebertran.

Blutuntersuchung: Beginn. Hämol. Vollst. Hämol. Res.-Br.

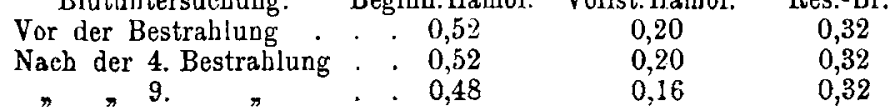

Nach der 4. Bestrahlung ist hier. noch keine Resistenzveränderung sichtbar. Sie tritt erst nach längerer Bestrahlung ein. Den Einfluss von Phosphor-Lebertran zu beurteilen ist nicht möglich. In der Literatur sind keine Angaben über einen solchen zu finden.

Fall 8. Soldat K., 21 Jahre, leidet an Bauchfelltuberkulose.

\begin{tabular}{|c|c|c|c|}
\hline Blut & Beginn. Hämol & Vollst. Hämol. & \\
\hline od & & 0,18 & \\
\hline & 0,46 & 0,18 & 0,28 \\
\hline & 0,50 & 0,14 & 0,36 \\
\hline
\end{tabular}

Fall 9. Herr M., 18 Jahre, leidet an Hilustuberkulose.

Blutuntersuchung: Beginn.Hämol. Vollst. Hämol. Res.-Br.

Vor der Bestrahlung . . . $0,50 \quad 0,0,20 \quad 0,30$

Nach der 6. Bestrahlung : . $0,48 \quad 0,16 \quad 0,32$

Fall 10. Herr Z., 19 Jahre, leidet an Lungentuberkulose.

Blutuntersuchung: Beginn.Hämol. Vollst.Hämol. Res.-Br.

Vor der Bestrahlung . . . $0,46 \quad 0,26 \quad 0,20$

Nach der 4. Bestrahlung : . $0,44 \quad 0,14 \quad 0,30$

Fall 11. Kind B., 14 Jahre, leidet an Spitzentuberkulose.

Blutuntersuchung: Beginn.Hämol, Vollst.Hämol. Res.-Br.

Vor der Bestrablung . . . $0,46 \quad 0,22 \quad 0,24$

Nach der 4. Bestrahlung : . $0,48 \quad 0,20 \quad 0,28$

Fall 12. Herr K., 25 Jahre, leidet an Tuberkulose.

Blutuntersuchung: Beginn.Hämol. Vollst. Hämol. Res.-Br.

Vor der Bestrahlung . . . $0,44 \quad 0,22 \quad 0,22$

Nach der 5. Bestrahlung : . $0,44 \quad 0,18 \quad 0,26$

Fall 13. Frl. S., 18 Jahre, leidet an Bronchialtuberkulose.

Blutuntersuchung: Beginn. Hämol. Vollst. Hämol. Res.-Br.

$\begin{array}{llcc}\text { Vor der Bestrablung . : : }: 0,40 & 0,20 & 0.20 \\ \text { Nach der 3. Bestrahlung : : } 0,40 & 0,20 & 0,20\end{array}$

Hier fehlt eine - aus äusseren Ursachen unterbliebene - spätere Untersuchung, die wohl eine Veränderung der Resistenz im Laufe der Bestrahlung erweisen würde.

Mit Ausnahme von 2 Fällen (6 und 13) ist also der Wert für die Maximumresistenz im Laufe der Bestrablung kleiner geworden. Die 
Minimumresistenz zeigt ein wechselnderes Verhalten. Ich komme später noch einmal darauf zurück.

Um nun die Möglichkeit, dass die gefundenen Veränderungen auf die jeweilige Krankheit oder Medikation zurückzuführen sei, ganz auszuschalten, habe ich noch, wio oben schon erwähnt, Untersuchungen an Gesunden vorgenommen. Sie sind ebenso bestrahlt worden, und die Untersuchungsmethode war gleichfalls dieselbe wie bei den zu therapeutischen Zwecken bestrahlten Personen. Das Ergebnis war bei allen gleichfalls eine bedeutende Resistenzerhöhung, die ebenfalls nach der 4. oder 5. Bestrahlung dentlich wird.

Ich lasse die Werte hier folgen:

Fall 14. Herr Kollege $\mathrm{H}$.

\begin{tabular}{|c|c|c|}
\hline $\begin{array}{l}\text { Blutuntersuchung: } \\
\text { Vor der Bestrablung }\end{array}$ & $\begin{array}{l}\text { Beginn. Hämol. } \\
\cdot \quad \cdot 0,42\end{array}$ & $\begin{array}{c}\text { Vollst. Hämol. } \\
0,24\end{array}$ \\
\hline Nach der 5. Bestrahlugg & 0,42 & 0,18 \\
\hline
\end{tabular}

Fall 15. Herr Kollege E.

Blutuntersuchung: Beginn.Hämol. Vollst. Hämol. Res-Br.

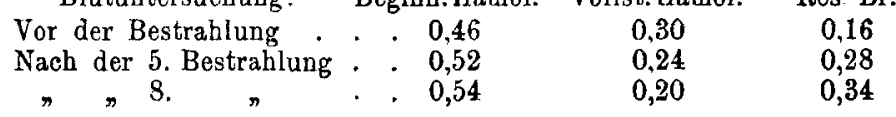

Fall 16. Herr Kollege R

Blutuntersuchung: Beginn. Hämol. Vollst. Hämol. Res.-Br.

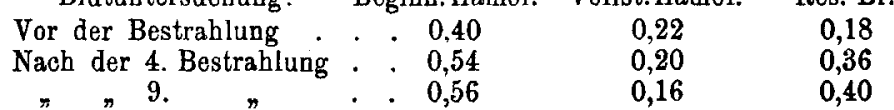

Fall 17. Herr Kollege $M$.

Blutuntersuchung; Beginn.Hämol. Vollst.Hämol. Res.-Br.

Vor der Bestrahlung . . . $0.42 \quad 0,24 \quad 0,18$

Nach der 5. Bestrablung: : $0,52 \quad 0,16 \quad 0,36$

Nach der 5. Bestrablung: $: \begin{array}{lll}0,42 & 0,16 & 0,26\end{array}$

Worauf die grosse Verschiedenheit der normalen Werte beruht, ist nicht zu sagen. Schon Bachrach, Gräfe, Keyes und Sachs, Port, Rywosch stellten ein verschiedenes Verhalten der Erythrozyten bei den verschiedenen Spezies und Individuen fest. Der Unterschied erweist sich auch durch die Bestrahlung nicht als beeinflussbar. Es findet nur eine annähernd parallele Verschiebung der Werte statt. Bei Fall 6 ist die niedrige Maximum- und hohe Minimumresistenz wohl aus der vor einigen Wochen stattgefundenen Bestrahlung zu erklären. Bei Fall 5, wo die frühere Bestrahlung länger zurückliegt ( $1 / 4 \mathrm{Jahr}$ ), ist ihr Einfluss nicht mehr deutlich nachweisbar. Ich gebe nebenstehend eine kurze Uebersicht in Tabellenform bei.

Das Ergebnis der Untersuchungen ist also bei Kranken und Gesunden eine Erböhung der Widerstandskraft der roten Blutkörperchen durch die Bestrahlung und zwar in zu therapeutischen Zwecken verwandter Stärke. Gleichzeitig zeigte sich auch ein gesteigertes Wohlbefinden der bestrahlten Personen.

Die Resistenzerhöhung ist schon nach der dritten bis vierten Bestrahlung, also nach einer Belichtungsdauer von ungefähr einer halben Stunde, deutlich. Sie bleibt bis zum Ende der Bestrahlung, ja noch längere Zeit danach bestehen, wie offenbar Fall 6 zeigt. 


\begin{tabular}{|c|c|c|c|c|c|c|c|c|c|c|}
\hline 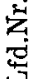 & Name & $\begin{array}{l}\text { Vor } \\
\text { Bestra }\end{array}$ & $\begin{array}{l}\text { der } \\
\text { lung }\end{array}$ & $\begin{array}{l}\text { Nach w } \\
\text { Bestrab }\end{array}$ & $\begin{array}{l}\text { cher } \\
\text { ing? }\end{array}$ & $\begin{array}{l}\text { Nach we } \\
\text { Bestrahl }\end{array}$ & $\begin{array}{l}\text { cher } \\
\text { ing? }\end{array}$ & Resi & enzbr & iten \\
\hline 1 & Erau R. K. & 0,40 & 0,20 & 4. 0,40 & 0,18 & 10. 0,46 & 0,18 & 0,20 & 0,22 & 0.26 \\
\hline 2 & Frau F. M. & 0,44 & 0,24 & 5. 0.44 & 0,20 & 10. 0,46 & 0,20 & 0,20 & 0,24 & 0.26 \\
\hline 3 & Frl. H. . & 0,40 & 0,20 & 3. 0,40 & 0,20 & 10. 0,46 & 0,14 & 0.20 & 0,20 & 0,32 \\
\hline 4 & Frau W. & 0,40 & 0,20 & 4. 0,42 & 0,16 & - & & 0,20 & 0,26 & - \\
\hline 5 & Herr S. . & 0,44 & 0.18 & 7. 0,48 & 0,16 & - & & 0,26 & 0,32 & - \\
\hline 6 & Soldat R. . & 0,54 & 0,12 & 4. 0,52 & 0,12 & 12. 0,46 & 0,12 & 0,42 & 0,40 & 0,34 \\
\hline 7 & Kind M. . & 0.52 & 0,20 & 4. 0,52 & 0,20 & 9. 0,48 & 0,16 & 0.32 & 0.32 & 0,32 \\
\hline 8 & Soldat $\mathrm{K}$. & 0,44 & 0,18 & 2. 0,46 & 0,18 & 7. 0,50 & 0,14 & 0,26 & 0.28 & 0,36 \\
\hline 9 & Herr M. & 0,50 & 0,20 & 6. 0,48 & 0,16 & - & & 0,30 & 0,32 & - \\
\hline 10 & Herr Z. & 0,46 & 0,26 & 4. 0,44 & 0,14 & - & & 0,20 & 0.30 & - \\
\hline 11 & Kind $\mathrm{B}$. & 0,46 & 0,22 & 4. 0,48 & 0,20 & - & & 0,24 & 0,28 & - \\
\hline 12 & Herr $\mathrm{K}$. & 0,44 & 0,22 & 5. 0,14 & 0,18 & - & & 0,22 & 0,26 & -- \\
\hline 13 & Frl. S. . & 0,40 & 0,20 & 3. 0,40 & 0,20 & - & & 0,20 & 0,20 & - \\
\hline 14 & Kollege H. & 0,42 & 0,24 & 5. 0,42 & 0,18 & - & & 0,18 & 0,24 & - \\
\hline 15 & Kollege $\mathrm{E}$. & 0,46 & 0,30 & 5. 0.42 & 0,24 & 8. 0,54 & 0,20 & 0,16 & 0,28 & 0,34 \\
\hline 16 & Kollege R. & 0,46 & 0,22 & 4. 0,54 & 0,20 & g. 0,56 & 0,16 & 0.18 & 0.36 & 0,14 \\
\hline 17 & Kollege $\mathrm{N}$. & 0,42 & 0,24 & 5. 0,52 & 0,16 & 9. 0,24 & 0.16 & 0,18 & 0,36 & 0,26 \\
\hline
\end{tabular}

Zu erwähnen sind uoch die Werte für die Minimumresistenz. Sie zeigen ein wechselndes Verhalten. Doch ist in den meisten Fällen eine deutliche Erhöhung derselben sichtbar. Fall 6, das ist der früher schon bestrahlte, zeigt eine deutliche konstante Abnahme der Minimumresistenz.

In der Erhöhung der Werte für die Minimumresistenz ist vielleicht die Uebereinstimmung mit der von Wanner gefundenen Resistenzverminderung im Hochgebirge, die nach seiner Meinung auf die natürliche Sonnenstrahlung zurückzuführen ist, zu finden. Doch scheint mir seine Ansicht nicht zu Recht zu bestehen, da er allein nur die Minimumresistenz untersucht und damit nur die eine Seite der Veränderungen berücksichtigt hat, während nach meiner Meinung von ausschlaggebender Bedeutung die Veränderung der Maximumresistenz ist.

Meine Untersuchungen, die hauptsächlich eine Veränderung der letzteren erweisen, lassen sich daher nicht zur Entscheidung der Frage heranziehen, ob die von Wanner gefundene Veränderung der Minimumresistenz ausschliesslich auf dem chemisch wirksamen Teil der Sonnenstrahlung beruht, oder ob nicht noch andere Faktoren des Höhenklimas dabei eine Rolle spielen. Meine Ergebnisse weisen aber jedenfalls dárauf hin, dass es nicht ausgeschlossen ist, dass die Hochgebirgssonne, besonders, wenn ihr der ganze Körper, wie es Rollier zu therapeutischen Zwecken tnt, eine längere Zeil ausgesetzt wird, eine Resistenzerhöhung. der Erythrozyten bewirkt.

Um noch schärferen Einblick in die Verhältnisse der Resistenzveränderung zu gekommen, habe ich ausserdem noch zwei Meerschweinchen bestrahlt. Einerseits hoffte ich das Verhalten der Minimumresistenz noch genauer verfolgen zu können, andererseits wollte ich die Wirkung von sehr starken Bestrablungen beobachten, wodurch vielleicht eine Analogie zu der von den dänischen Forschern in vitro festgestellten Strahlenhämolyse zu finden war.

Die Versuchsanordnung blieb die gleiche. Jedoch verwandte ich kein Glaubersalz, weil ich, ohne vorzeitige Gerinnung befürchten zu 
müssen, mittelst Kapillare das aus dem Ohr des Meerschweinchens tropfenweise gewonnene Blut dirckt in die Röhrchen mit den verschiedenen Salzkonzentrationen bringen konnte, was aus äusseren Gründen früher nicht gut angängig war. Die Tiere wurden nach Rasieren des Rückens jeden Tag einer Bestrahlung unterworfen, die ich bei dem einen (braunen) täglich um 5 Minuten, bei dem andern (schwarzen) um 10 Minuten steigerte.

Die Tiere wurden vor der Enthaurung, dann $3 \mathrm{mal}$ vor der Bestrahlung untersucht, um normale Ausgangswerte zu bekommen. Während der Bestrahlung wurden sie täglich 3 mal untersucht.

Es konnte nun absolut keine deutliche Veränderung der Resistenz festgestellt werden. Die geringen Schwankungen nach oben und unten liegen innerhalb der Fehlergrenzen.

Ich bestrahlte nun die Tiere plötzlich $1 \frac{1}{2}$ Stunde lang. Auch jetzt blieb die Resistenz unverändert, während sich das Blutbild deutlich änderte. Kand. med. Baumann wird darüber berichten. Die Tiere nahmen ab, vor allem das braune. Nach der dritten $1 \frac{1}{2}$ stündigen Bestrahlung starb das braune Meerschweinchen. Auch die Blutuntersuchung direkt vor dem Tode blieb ohne Ergebnis. Die Sektion ergab schwere Veränderungen der inneren Organe, die als Folge der Strahlenwirkung anzusehen sind. Ich gebe die Werte der einzelnen Untersuchungen in Tabellenform bei.

$\begin{array}{lll}\text { 17. 11. } & 0,48 & 0,30 \\ \text { 18. 11. } & 0,46 & 0,28 \\ \text { 19. 11. } & 0,48 & 0,28 \\ \text { 20. 11. } & 0,50 & 0,32 \\ \text { 21. 11. } & 0,48 & 0,28 \\ \text { 22. 11. } & 0,48 & 0,28 \\ \text { 23. 11. } & 0,48 & 0,30 \\ \text { 24. 11. } & 0,46 & 0.30 \\ \text { 26. } 11 . & 0,50 & 0,30\end{array}$

Meerschweinchen I (braun), $350 \mathrm{~g}$.

\begin{tabular}{|c|c|c|c|c|c|}
\hline Ras & eren & 0,44 & 0,30 & 0,46 & 0,3 \\
\hline & Min. & 0,50 & 0,30 & 0,50 & \\
\hline 10 & $\eta$ & 0,64 & 0,28 & 0,50 & 0,2 \\
\hline 15 & $"$ & 0,46 & 0,30 & 0,46 & 0,3 \\
\hline 20 & $m$ & 0,48 & 0,28 & 0.48 & \\
\hline 25 & & - & - & 0,48 & 0,2 \\
\hline $11 / 2$ & Stdd. & 0,50 & 0,28 & $?$ & \\
\hline $11 / 2$ & 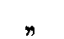 & - & - & 0,48 & 0,3 \\
\hline & & & & 0,52 & \\
\hline
\end{tabular}

Lampenabstand $1 \mathrm{~m}$

Meerschweinchen II (schwarz), $420 \mathrm{~g}$.

\begin{tabular}{|c|c|c|c|c|c|c|c|c|}
\hline 17. 11. & 0,52 & 0,34 & Rasieren & 0,46 & 0,32 & 0,46 & 0,32 & Lampenabstand $1 \mathrm{~m}$ \\
\hline 18. 11 . & 0,46 & 0.34 & 10 Min. & 0,48 & 0,34 & 0,50 & 0,34 & - \\
\hline 19. 11 . & 0,48 & 0,36 & $20 \%$ & 0,50 & 0,36 & 0,48 & 0,36 & - \\
\hline 20. 11 . & 0,48 & 0,36 & 30 & 0,48 & 0.34 & 0,46 & 0,34 & $420 \mathrm{~g}$ \\
\hline 21. 11. & 0,48 & 0,36 & 40 & 0,46 & 0,34 & 0,46 & 0,3 & - \\
\hline 22. 11 . & 0,48 & 0,34 & 50 & - & - & 0,48 & 0,34 & $440 \mathrm{~g}$ \\
\hline & 0,50 & 0,36 & & 0,48 & 0,34 & 0,48 & 0,36 & Lampenabstand $45 \mathrm{~cm}$ \\
\hline 24. 11 . & 0,48 & 0,36 & $11 / 2$ Std. & - & - & 0,48 & 0,36 & $410 \mathrm{~g}$ \\
\hline 6. 11 . & 0,48 & 0,36 & $11 / 2 \pi$ & - & - & $\ldots$ & - & \\
\hline
\end{tabular}

Wie ist das Fehlen einer Resistenzänderung zu erklären? Vielleicht beruht das darauf, dass die Unterschiede zu klein sind, um mit den relativ groben Untersuchungsmitteln erfasst zu werden, sind ja auch beim Menschen die Ausschläge verhältnismässig nur gering. Auf der andern Seite haben schon mehrere Untersucher z. B. M. Levy betont, dass Strahlenwirkungen bei Tieren nicht in jedem Falle auf den Mensehen oder umgekehrt bezogen werden können, sind ja selbst bei den einzelnen Tiergattungen Abweichungen vorhanden. 
Wie ist nun beim Menschen die Beeinflussung der Resistenz durch die ultravioletten Strahlen zu verstehen?

Die Erhöhung der Maximumresistenz ist vielleicht damit zu erklären, dass durch die Erhöhung der ganzen Stoffwechselvorgänge auch ein Reiz auf das Knochenmark ausgeübt wird, der dasselbe zu reichlicher Abgabe von jungen lebenfrischeren und daher widerstandsfähigeren (Sattler, Krasny) Erythrozyten in Blut anregt.

Wie kommt aber das gleichzeitige Steigen der Minimumresistenz zu Stande? Sie als bloss unberechenbare Schwankungen, wie Cohnreich meint, zu deuten, dazu tritt nach meiner Meinung diese Erscheinung zu regelmässig und deutlich auf. Vielleicht liegt hier doch etwas Analoges vor, wie es Schmidt-Nielsen berichtet hat (s. o.). Nach Bering filtriert die Epidermis die äussersten ultravioletten Strahlen weg und die für die Fermente toxischen Strahlen kommen nicht ans Blut heran. Die blauvioletten und inneren ultravioletten Strahlen dagegen gehen durch die Epidermis hindurch und werden von dem „roten Blutschirm ${ }^{4}$ der Hautkapillaren aufgefangen und absorbiert. Das Blut wirkt als Lichtfilter, indem es nach Busck die ultravioletten Strahlen zu 99 pCt. absorbiert. Durch diese aufgenommene grosse Strahlenenergie müssen die Blutzellen zweifellos auf irgend eine Weise beeinflusst werden, wie man es auch schon für andere Zellen nachgewiesen hat.

Man wird wohl nicht fehlgehen, die Resistenzveränderung als Ausdruck der Strahlenwirkung anzusehen und zwar so, dass zunächst eine leichte Störung im osmotischen Gleichgewicht auftritt, die ihren nachweisbaren Ausdruck in der erhöhten Minimumresistenz, in gewissem Sinne also einer direkten Schädigung der Erythrozyten findet (die später offenbar wieder auf ihre alten Werte zurückkehrt), dass dann aber das Knochenmark reaktiv mit der Abgabe jüngerer und widerstandsfähigerer Zellformen antwortet, durch die dann die Vertiefung der Maximumresistenz bedingt ist, d. h. also zum Schluss eine Erhöhung der Resistenzbreite in Erseheinung tritt. Man könnte also von „Aktion und Reaktion" gegenüber den ultravioletten Strahlen sprechen. Vielleicht ist hiermit eine annehmbare Erklärung für meine Befunde gegeben.

Verschiedenartige Reize können Veränderung der Resistenz hervorrufen. Zu diesen Reizen gehören also auch die ultravioletten Strahlen. Die Ansicht aber, dass dieselben wie Bordier angegeben hat, keinen Einfluss auf das Blut haben, kann nicht aufrecht erhalten werden.

\section{Zusammenfassung.}

1. Die von Wanner angegebene Resistenzverminderung durch natürliche Sonnenbestrahlung des Körpers ist wahrscheinlich nicht als einzige Wirkung der ultravioletten Strahlen anzusehen. Denn Bestrahlung mit ultraviolettem Licht in therapeutischen Dosen ruft beim Menschen neben einer Erhöhung der Minimumresistenz eine Vertiefung der Maxinumresistenz hervor, die nach der 4.-5. Bestrahlung nachweisbar wird und noch längere Zeit nach derselben anhält. 
2. Die damit gegebene Erhöhung der Resistenzbreite ist vielleicht als Aktion und Reaktion gegenüber den ultravioletten Strahlen aufzufassen, im Sinne direkt schädigender und reaktiv reparatorischer Vorgänge.

3. Beim Meerschweinchen ist durch dio Bestrahlung eine Resistenzveränderung nicht zu erzielen.

Es ist mir zum Schluss eine angenehme Pflicht, Herrn Prof. Dr. Ziegler für die Zuweisung der Arbeit und das tätige Interesse, sowie Herrn Oberarzt Dr. Königsfeld für seine Unterstützung dabei meinen herzlichsten Dank zu sagen.

\section{Literaturverzeichnis.}

Aggazotti, Arch. ital. de Biol. 1907. Vol. 47. Zit. nach Cohnheim. Bach, Deutsche med. Wochenschr. 1911. Nr. 9. - Bachrach und Grafe, Arch. f. Hyg. 1909. Bd. 70. Zit. nach Cohnheim. - Baer u. Engelsmann, Deutsches Arch. f, klin. Med. Bd. 112. - Bering, Med.-naturwissenschaftl. Arob, 1908. Bd.1. - Derselbe, Münchener med. Wochenschr. 1912. Nr. 51. - Bering u. Meyer, Strahlentherapie. Bd. 1 u. Bd. 3. - B orner, Strahlentherapie. Bd.5. - Bernhard, Strahlentherapie. Bd. 8. - Bordier, Strahlentherapie. Bd. 2. - Bürker, Pflüg. Arch. 1904. Bd. 102. S. 36. Zit. nach Cobnbeim. - Derselbe, Verhandl. d. deutsch. Physiol. Geselisch. Zentralbl. f. Physiol. 1911. Nr. 23. Zit. nach Cohnheim. - Busck, Berliner klin. Wochenschr. 1907. Nr. 28. - Derselbe, Mitteil. a. Finsen's med. Lichtinst. Bd. 4. - Charlton, Med. Diss. Berlin 1916. - Cohnheim, Asher-Spiro Physiol. 1912. Bd. 12. - Cobnheim u. Weber, Arch. f. klin. Med. Bd. 110. - Cohnreich, Fol. haematol. 1913. Bd. 16. - Deetjon, Zeitsohr. f. physiol. Chem. 1909. Bd. 63. H. 1. Zit. nach Cobnheim - Dorno, Licht und Luft im Hochgebirge. 1911. Braunschweig. - Douglas, Journ. of. Physiol. 1910. Bd. 40. S. 454. Zit. nach Cohnheim. - Dörig, Kollmer, Reichel, Physiol. Ergebn. d. Monte-Rosa-Expedition. Denkschr. d. Wiener Kaiserl. Akad, d. Wissensch. Mathem.-naturw. Kl. Bd. 86. Zit. nach Cohnheim. - Durig, Zuntzu.Neuberg, Biochem. Zeitschr. Bd. 72. - Dreyer u. Hansen, Comptes rendues. - Egger, Arch. f. exp. Pathol. u. Pharmakol. 1897. Bd. 39. Zit. nach Cohnhoim. Galeotti, Arch. ital. de Biol. 1904. Vol. 41. p. 80. Zit. nach Cohnheim. Haldane u. Douglas, Journ. of. Physiol 1909. Bd. 38. S. 420. Zit. nach Cohnheim. - Hamburger, Osmot. Druck u. Ionenlehre. Wiesbaden 1902. - Hasselbach, Biochem.Zeitschr. 1909. Bd.19. - Derselbe, Stralentherapie. Bd.2. - H as selbach und Lindhardt, Biochem. Zeitschr. Bd. 68 u. 74. - Dieselben, Skand. Arch. f. Physiol. 1911. Bd. 25. - Hausmann u. Mayerhofer, Biochem. Zeitschr. Bd. 72. - Herte 1, Zeitscbr. f. allg. Physiol. 1907. Bd. 6. - Höber, Physikal. Chem. d. Zellen u. Gewebe. Zit. nach Port. - Itami u. Pratt, Biochem. Zeitschr. Bd. 18. - Janowsky, Klin. Wochenschr. Petersburg 1887-89. Zit. nach Cohnreich. - Jaquet u. Stähelin, Arch. f. exp. Patholog. a. Pharmak. Bd. 46. Zit. nach Cohnheim. - Jesionek, Strahlentherapie. Bd.7. - Krasny, Fol. haematologica. 1913. Bd. 16. - Kabn, Münchener med. Wochenschr. 1907. S. 1712. Kyos u. Sachs, Berliner klin. Wochensehr. Zit. nach Cohnroich. - Lang, Zeitschr. f. klin. Med. 1902. Zit. nach Cohnbeim. - Laquer, Deutsches Arch. f. klin. Med. Bd.110. - Lery, Strablentherapie. Bd. 7. - Derselbe. Ebenda. Bd.9. - von Limbeck, Grundriss einer klin. Pathol. d. Blutes. Zit. nach Cohnreich. —Lovy, Zeitschr. f. allg. Physiol. 1911. Bd.13. - Moss 0, Arch. ital. de biol. 1905. 
458 von Robden, Einfluss d. Queeksilberquarzlampenlichts auf die Resistenz usw.

Vol.43. p. 355. Zit, nach Cobnheim. - Derselbe, Ebenda. 1887. Vol. 1. p. 7. Neuberg, Biochem.Zeitschr. Bd. 13, 17, 29, 39. - Pinkuss obn, Strahlentherapie. 1913. Bd. 3. - Port, Arch. f. exp. Pathol, u. Pharmakol. Bd.69. - Quincke, Arch. f. d. ges. Physiol. 1894. Bd. 57. - Rollier, Strahlentherapie. Bd. 4. - Derselbe, Deutsche Zeitschr. f. Chirurg. Bd. 116. - Ribierre, Fol. haematolog. 1905. Bd. 2. - Rosentbal, Ebenda. Arch.10. - Rywosch, Pfüg. Arch. 1907. Bd. 116. Zit. naoh Cohnreich. - Sattler, Fol. haemotol. 1910. Bd. 9. - Schmidt-Nielsen, Nyt. Magaz, for Naturvidenskaft. 1909. - Dieselben, Mitteil. a. Finsen's med. Lichtinst. 1906. Bd. 10. - Stäubli, Oberengadiner Festschr. 1911. Zit. nach Cohnheim. - Strasser u. Noumann, Med. Klin. 1909. Nr. 34. - Thiele, Med. Diss. Berlin 1916. - Wanner, Deutsche Zeitschr. f. Chir. Bd. 116. - Waltscheff, Med. Diss. Berlin 1915. - Weihrauch, Deutsche med. Wochenschr. 1913. Nr. 18. - Wernseheid, Med. Diss. Bonn 1918. - Zuntz, Lery, Müller und Caspary, Höhenklima und Bergwänderungen. Bong \& Co. Berlin-Leipzig 1906. Zit. nach Cohnheim. 